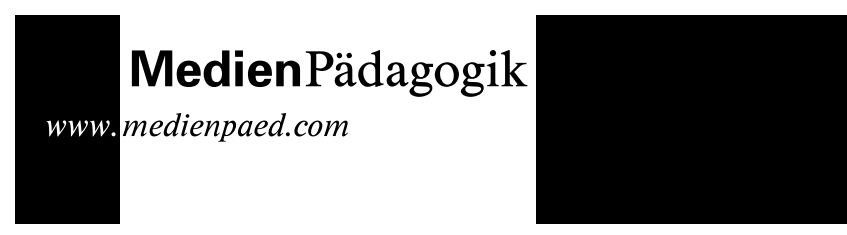

Stefan Krause und Rolf-Dieter Kortmann

30.10 .2002

\section{Standardisierung im E-Learning oder Vom schleichenden Untergang} der Didaktik

\section{Einleitung}

«The SCORM course meta-data specification is based upon the IMS Learning Resource Meta-data Information Model. However, the IMS metadata specification is itself derived from the IEEE LTSC Learning Object Meta-data (LOM) Draft Standard, ver 6.1 (2001-4-18)» (SCORM Resources, 2001).

Bislang haben die Standardisierungsbestrebungen im Bereich des ELearnings lediglich eine nahezu unüberschaubare Anzahl an Abkürzungen und Akronymen hervorgebracht. Doch verglichen mit der Geschichte der International Organization for Standardization (ISO) oder des Deutschen Instituts für Normung e. V. (DIN) handelt es sich noch um junge Ansätze. Tatsächlich existieren bereits verschiedene Spezifikationen, die darauf angelegt sind, eines Tages zum Standard zu werden. Von einer Standardisierung im eigentlichen Sinne ist man aber noch weit entfernt.

Während der Sinn technischer Standards unmittelbar einleuchtet - hat doch beispielsweise die Einführung des «Rich Text Format» (RTF) durch Microsoft den Datenaustausch und die tägliche Zusammenarbeit unterschiedlicher Textverarbeitungsprogramme erheblich erleichtert - stellt die Standardisierung von Inhalten nicht nur ein methodisches Problem dar.

\section{Standardisierung von Inhalten}

Die Standardisierung von Inhalten (z. B. durch die IEEE Working Group 1484.12) basiert ebenso wie die der technischen Spezifikationen auf der Vorstellung, den Austausch von Daten zu vereinfachen und Interoperabilität von Lernplattformen herzustellen. Lediglich die Art der auszutauschenden Daten ist eine andere: Es handelt sich nicht mehr um Daten- formate, sondern um so genannte Lernobjekte. Diese sind als eigenständige, in sich geschlossene Lerneinheit mit Inhalten, Übungen und Tests definiert (Daten + Dokumentation, 2002). Sie können in verschiedenen Kursen und Seminaren eingesetzt werden, wodurch schon eine ihrer wichtigsten Eigenschaften angedeutet ist: Sie sind wieder verwendbar. Die Vorteile sind klar ersichtlich: Kleine, in sich abgeschlossene Einheiten, die getrennt von Datenformaten und durch Metadaten (bspw. LOM, IEEE P1484.12/D6.1, 2001) beschrieben vorliegen, sind wirtschaftlich. Sie können ständig aktualisierbar in jedwede Plattform eingebunden werden und der Aufwand, gleiche Inhalte ein zweites Mal herzustellen, wird reduziert.

Die Grösse dieser Objekte, welchen Umfang und welchen Komplexitätsgrad die ihnen innewohnende Struktur hat, ist nicht festgelegt und differiert stark. Mittelfristig wir sie sich aber auf einen Wert einpendeln, bei dem der wirtschaftliche Vorteil der Wiederverwertbarkeit noch nicht durch erhöhte Kosten durch Katalogisierung und Verwaltung aufgehoben wird. Dieser Umstand, nicht didaktische Überlegungen, wird auch die vollständige Atomarisierung dieser «learning objects» verhindern.

Durch ihre Granularität passen sich Lernobjekte gut in den Hochschulalltag ein. Sie liegen, ähnlich wie Vorlesungsfragmente und Diasammlungen, in zentralen Pools bereit, können dort leicht abgerufen, in den eigenen Unterricht integriert oder zur digitalen Veröffentlichung mit anderen Lernobjekten auf Lernplattformen kombiniert werden. Dieser Trend ist auch in Deutschland $\mathrm{zu}$ beobachten. In einer Zusammenfassung eines nationalen Workshops beschreibt beispielsweise Fromann (2001) den dort formulierten Konsens mit folgenden Worten: «Zahlreiche Fürsprecher fand die Position, statt didaktisch komplexer Lerneinheiten lediglich Medienelemente in hoher Granularität und breiter Verfügbarkeit zu entwickeln. Als besonderer Vorteil wurde hier der Zugriff der Lehrenden auf einzelne Bausteine gesehen. Dies sei vor dem Hintergrund der Belastung in Lehre und Forschung ein pragmatischer Weg zur effizienten Nutzung der Möglichkeiten der Neuen Medien in der Lehre» (S. 1f, s. a. Kindt, 2001). Bleibt eine Frage: Verdienen diese «Informationshäppchen» tatsächlich die Bezeichnung «Lernobjekt»? Angesichts der vielfach geführten Diskussion zu den neuen didaktischen Möglichkeiten der computerbasierten Medien kann weder der Hinweis, dass es «zu lernende Objekte» sind, noch, dass es Objekte einer Lernumgebung sind oder dass die Inhalte dieser Objekte Lerninhalte darstellen, genügen. Zu unmittelbar ist mit dieser Begrifflich- 
keit der Gedanke der Lernförderlichkeit assoziiert, als dass die Benennung nur pragmatische Gründe haben sollte: Sie verkörpert geradezu den Anspruch des Objekts, am Lernprozess beteiligt zu sein. Inwieweit Lernplattformen, die technische Heimat der Lernobjekte, aber per se lernwirksam sind, bleibt häufig offen. Die hypermedialen Ansätze der zweiten Stunde (kognitivistische Modelle mit behavioristischen Einschlägen) waren durch die technikeuphorische Annahme geprägt, dass sich durch neue Repräsentations- und Darstellungsmöglichkeiten von Informationen sowie durch Möglichkeiten des selbst gesteuerten interaktiven Zugriffs und Umgangs mit Informationen quasi automatisch Vorteile für den Wissenserwerb ergeben (Tergan, 1998). Dabei wurde verkannt, dass auch bei Multimedia- und Hypermedia-Systemen das Potenzial zur Unterstützung von Lernprozessen erst durch Aktivierung konstruktiver kognitiver Verarbeitungsprozesse erschlossen werden muss.

Nach der konstruktivistischen Wende mit einer Ausdifferenzierung der didaktischen Ansätze besteht durch die Bemühungen zur Standardisierung und den damit verbundenen Aufschwung der Lernobjekte wieder die Gefahr des Rückfalls in die Zeit, in der neue Medien nur zur Vermittlung von demjenigen Wissen eingesetzt wurden, das durch Kriterien wie Verstehen, Behalten und wortgetreuer Wiedergabe überprüft werden kann. Didaktische Modelle, die zu explizieren versuchen, warum und welche ihrer Konstruktionsaspekte lernförderlich sind, geraten auf diese Weise ins Hintertreffen. Zu verlockend ist es, auf dem Boden standardisierter Module langfristig kostengünstig und mit früher ungeahnten Distributionsmöglichkeiten durch das «Netz aller Netze» die für relevant erachteten Inhalte in der Form von Informationssammlungen bereitzustellen.

So wurde durch die Bemühung der Standardisierung, die Hand in Hand mit der Modularisierung von Lerninhalten ging bzw. zu dieser führte, schleichend einem neuen (oder, instruktionspsychologisch gesehen, sehr alten) Paradigma der Weg bereitet. Es besteht zunehmend die Tendenz, lediglich Informationen in (Hyper-)Textform auf Lernplattformen zusammenzustellen, die das nach den Vorstellungen der Autoren zu erlernende Wissen darstellen. Angereichert werden diese Textpassagen mit multimedialen Komponenten oder Multiple-Choice-Aufgaben, die aber häufig mehr der Auflockerung dienen als dass ihr Einsatz didaktisch begründet wäre (Schulmeister, 2001). In einer Einführung in das «... instructional technology concept known commonly as the 〈learning object»» beschreibt Wiley (ebd.) die Vorteile der Modularisierung, allerdings nicht ohne immer wieder den Einsatz einer wieder einmal nicht näher spezifizierten Didaktik gleichsam formelhaft zu beschwören (Wiley, 2001): «... there must be concentrated effort made to understand the instructional issues inherent in the learning objects notion» (S. 29). Auch Schulmeister sieht in den Initiativen zur Formulierung von Metadaten und Standards keine Garantie dafür, «dass die Bildungsinteressenten dadurch ein Stück schlauer werden und sich informierter entscheiden können. Das geben diese Kriterien nicht her. An die eigentlichen pädagogischen Prinzipien virtueller Lehre hat sich bisher keines der internationalen Gremien gewagt» (Schulmeister, 2001, S. 22).

Möglicherweise rücken andere Argumente für den Einsatz standardisierter digitaler Lehre in den Vordergrund. Üblicherweise werden zusätzliche Vorteile der internetbasierten Medien mit Schlagworten wie «Zeit- und ortsunabhängiges Lernen», «individuelles Lernen», «Motivationssteigerung», «Qualitätssteigerung», «Beschleunigung des Wissenstransfers», «permanente Aktualisierung», «Kostenreduktion», «Nachhaltigkeit, «Flexibilität», «Vermittlung von Medienkompetenz» und «Kommunikation» umschrieben. Auch wenn der Mehrwert nicht aller dieser Begriffe allein auf der Nutzung der neuen Medien per se basiert, ist die Begründetheit auch anderer Aspekte als die der Lernförderlichkeit nicht in Abrede zu stellen. Doch muss die Priorität klar benannt werden, anstatt durch den allgegenwärtigen Zusatz «didaktisch» eine wie auch immer geartete Unterstützung des Lernprozesses zu suggerieren, die de facto in vielen Ansätzen nicht existiert. Der Nachweis, dass die interoperativen Informationsmodule tatsächlich einen Beitrag zum Wissenserwerb darstellen, muss für jede Spielart der Lernplattformen neu geführt werden. Allein der Name ist noch nicht Programm.

\section{Konstruktion einer lernwirksamen Anwendung - der theoretische \\ Hintergrund}

Die Konstruktion einer lernwirksamen Anwendungen setzt die Identifikation lernwirksamer Momente voraus. Gleichzeitig muss für eine konsequente Umsetzung eine Reduktion auf bestimmte Prinzipien vorgenommen werden. Die Zeiten sind vorbei, in denen in einem multimedialen Overkill alle Interessen der verschiedenen Lernertypen mit einer einzigen Anwendung zu erschlagen versucht wurden. 


\section{Exkurs: Medizinische Expertise}

Schon früh wurde in der Expertisenforschung der Blick auf den Sachverhalt gelenkt, der für die Ausführungsgüte von Aufgaben unterschiedliche Wissensarten von Bedeutung ist. Wichtig wurde in diesem Zusammenhang vor allem die Unterscheidung von dem deklarativen, in propositionalen Netzwerken organisierten Faktenwisse einerseits und dem Handlungswissen, das in der Literatur auch als prozedurales Wissen bezeichnet wird und in sogenannten Produktionssystemen organisiert ist anderseits (Anderson, 1989).

Klinische «Anfänger»- Medizinstudenten im klinischen Abschnitt und im Praktischen Jahr - zeichnen sich häufig durch ein hohes Detaillwissen aus, versagen aber, wenn sie unter Zeitdruck und bei unsicherer Datenlage Entscheidungen treffen müssen. Gerade diese Kombination ist aber ein Spezifikum der ärztlichen Situation. Woran liegt es, dass das erworbene Wissen nicht entsprechend anwendbar ist, oder anders gefragt: Was unterscheidet den medizinischen Experten vom Novizen? Nach Groen \& Patel (1991) sind es vor allem folgende drei Kriterien. Zum einen ist der von den Experten entwickelte Weg, die in der Problemlösesituation enthaltenen Informationen im Arbeitsgedächtnis (Kurzzeitgedächtnis) zu repräsentieren, hocheffizient. Während die Aufnahmefähigkeit normalerweise auf $7+/$ - 2 Items begrenzt bleibt, gelingt es ihnen, diese Kapazität (beispielsweise durch chunking) zu vervielfachen. Zum anderen ist das Wissen im Langzeitgedächtnis als Satz hochspezifischer, domänbezogener Operatoren («set of production rules ${ }^{`}$ ) gespeichert. Die einzelnen Informationseinheiten bestehen nicht mehr aus Informationen über etwas, sondern aus «wenn-dann-Regeln», die vorgeben, wie in einer Situation zu handeln ist, wenn die Conditio, der «wenn»-Teil der Regel, erfüllt ist. Der Grad der Expertise wird entsprechend der Anzahl der hochspezifischen, domänbezogenen Regeln bestimmt. Und schliesslich bedienen sich Experten der Vorwärtssuche (Analyse der Datenlage durch Mustererkennung) als Problemlösestrategie. Von dieser datenorientierten Vorgehensweise weichen sie nur bei Unsicherheit bzw. in Situationen, in denen sie nur wenig Erfahrung haben, ab. In diesen Fällen verwenden sie die Rückwärtssuche, eine als hypothesen-testende Strategie zu verstehende Vorgehensweise, die generell die Vorgehensweise von Novizen kennzeichnet ${ }^{1}$.

${ }^{1}$ Neben diesen drei Kriterien muss noch ein anderer Aspekt erwähnt werden: Das $5 / 10$
Für die medizinische bzw. klinische Ausbildung ist die Frage entscheidend, wie das Expertendenken gelehrt werden kann. Versuche, dieses Wissen zu extrahieren, um es vermittelbar zu machen, scheiterten. Das Expertenwissen ist nicht nur subjektiv konstruiert und dadurch nur subjektiv verfügbar, auch der Prozess der Konstruktion auf der Basis deklarativen Wissens als solcher scheint Voraussetzung für die spätere Verfügbarkeit von Erfahrungswissen zu sein.

Nach Kolodner (1983) basiert der Erwerb von Expertise auf der reflexiven Anwendung von Wissen. Lernen wird dabei zum einen durch die Generalisierung über Episoden hinweg, zum anderen durch die Analyse von Fehlern initiiert. Die sich bildenden Schemata, die «illness scripts» (Schmidt et al., 1990) werden auf diese Weise verfeinert, von Fehlern bereinigt und somit in konkreten Situationen (z. B. Diagnose von Patienten) zunehmend anwendbar. Oder wie Gruber (1999) in bezug auf das klinische Problemlösen formuliert: «Die [...] relevanten Probleme sind zumeist schlecht definiert, selten linear, immer in situationale Kontexte eingebettet und kaum zu Beginn des Problemlöseprozesses schon vollständig ausgearbeitet. Das zur Lösung solcher Probleme erfolgversprechendste Wissen ist das Erfahrungswissen bzw. «tacit knowledge». Dieses Wissen entsteht in der Regel nicht durch explizite Unterweisung, sondern durch die konstruktive Auseinandersetzung Lernender mit selbst erfahrenen, authentischen Problemsituationen, sofern eine aktive Verarbeitung der dabei relevanten Informationen stattfindet» (S. 37).

\section{Konstruktion einer lernwirksamen Anwendung - praktische Umsetzung am Beispiel der Klinikumssimulation PROMETHEUS}

Ein entscheidender Aspekt von Simulationen ist der Erwerb von Handlungswissen durch die Anwendung bestehenden oder erworbenen Faktenwissens. Das Ergebnis dieses Wissenskonstruktionsprozess - ein Wissen, das eher in Form einer Ansammlung von Handlungsregeln vorliegt als in kausalen, propositionalen Netzwerken - kann nicht gelehrt, sondern nur aufgrund eigener Handlung konstruiert werden. Es ist das Wissen, das den

Können des Experten gilt als subjektives Wissen. Das durch Erfahrung prozeduralisierte, ehemals deklarative Wissen ist nicht mehr als objektives «Weltwissen» verfügbar, da es durch einen idiosynkratischen

Wissenskonstruktionsprozess gebildet wurde und nur noch in einer individuellen, nicht spezifizierbaren Weise mit dem urprünglichen Wissen korrespondiert. 
Experten vom Anfänger unterscheidet und das der Unerfahrene vermisst, wenn er an Aufgaben scheitert, die er aufgrund seines Informationsstandes - dem Stand seines deklarativen Wissens - eigentlich meistern müsste. Günstigerweise wird dieses Wissen im konkreten Umgang mit realen Situationen erworben. Angesichts der ethischen Probleme, Lernenden in gefährlichen Situationen Handlungsfreiheit zu geben, um ihnen das «learning by doing» zu ermöglichen, stellen realitätsnahe, computergestützte Simulationen eine wertvolle Alternative dar, die Genese von Prozessen zu unterstützen, die normalerweise durch reale Handlungen initiiert werden sollen. Simulationen schaffen somit die Möglichkeiten, durch Anwendung deklarativen Wissens Handlungswissen $\mathrm{zu}$ konstruieren und dadurch in komplexen Situationen handlungsfähig zu werden, ohne dass bei Fehlhandlungen Schaden in der tatsächliche Welt entsteht. Damit wird ein Aspekt des Wissenserwerbs fokussiert, der durch die neuen Medien besonders gewinn bringend realisiert werden kann und für dessen Erwerb nur unzureichend Alternativen bestehen.

Inhaltlicher Schwerpunkt des BMBF-geförderten Projekts PROMETHEUS (www.prometheus.uni-tuebingen.de) ist demzufolge eine Klinikumssimulation, in deren Mittelpunkt der Prozess der ärztlichen Entscheidungsfindung steht. Der Studierende untersucht virtuelle Patienten mit dem Ziel, durch das Anwenden klinischer Vorgehensweisen Diagnosen zu erarbeiten. Hierbei wird vorhandenes Wissen (das bis dato «träge Wissen»; Gräsel et al, 1993) so auf den Patienten bezogen, dass eine stimmige Erklärung für das vorliegende Krankheitsbild gefunden werden kann. Beim Erarbeiten der Diagnosen wird vorhandenes bzw. für die Lösung eines Falles erworbenes Wissen an besondere Sachverhalte angepasst, wobei es sich durch diese Konstruktionsprozesse verändert. Es findet «Lernen durch Problemlösen» statt.

Simulierte, virtuelle Realitäten mit ihrer charakteristischen Kombination von räumlicher Situierung und interaktiver Nutzbarkeit stellen eine förderliche Lernumgebung dar, die fundamentale Vorteile gegenüber traditionellen Lernmedien - also Texten, Illustrationen, Diagrammen oder Filmen haben und somit der Forderung nach einer expliziten Didaktik der Neuen Medien entsprechen. Aus Sicht der kognitiven Medienpsychologie sind mit solchen Eigenschaftsunterschieden eine Reihe von Konsequenzen für Prozesse der Informationsverarbeitung und des Wissenserwerbs beim Lerner verbunden, die eine vertiefte, handlungsorientierte Abspeicherung neuer Inhalte weit stärker ermöglicht (Schwan \& Buder, im Druck). Ist die virtuelle Realität immersiv genug gestaltet, kommt es durch Speicherung der neuen Inhalte in neuronale Netzstrukturen, die ähnlich zu denjenigen sind, in der die zukünftige reale Situation repräsentiert wird, zusätzlich zu zahlreichen Assoziationseffekten, die Qualität und Geschwindigkeit des Dekodierens erhöhen - das Gelernte steht somit in dem späteren Anwendungskontext schneller zur Verfügung (Anderson, 1989).

Ein weiterer Aspekt in der didaktischen Konzeption ist die Frage, wie die intrinsische Motivation geweckt und gezielt eingebunden werden kann. Lernen Kinder noch häufig aus Eigeninteresse oder «um der Sache willen», wird diese lernförderliche Haltung zumeist in der Schule durch extrinsische Motivationen (z. B. Noten, Antizipation des zukünftigen Selektionsvorteils etc.) korrumpiert. So ist es die Aufgabe von Lernumgebungen, das Handeln der Lernende aus eigenem Antrieb (Interesse, Neugier, kurz: Die intrinsiche Motivation) zu wecken. Hierzu wird bei PROMETHEUS das Konzept «Goal Based Scenario' (Schank, 1994) eingesetzt, bei dem das Handeln und Lernen auf dem Weg zu einem bestimmten Ziel stattfindet. Das Ziel ist, die richtige Diagnose zu finden, neue Informationen und Handlungen werden mit Orientierung auf das Lösen des Patientenfalls verarbeitet. Sieht man des Gedächtnis als ein «Abfallprodukt denkerischer Tätigkeit», entsteht eine lernwirksame Lernumgebung durch den Anreiz zum Denken. Oder wie Benjamin Franklin bemerkte:

Tell me and I forget,

Teach me and I remember,

Involve me and I learn.

B. F.

\section{Fazit}

Informationen per se, die zunehmend häufig als standardisierte Textbausteine unter dem Dach verschiedener Lernportale angesammelt werden, leisten nur noch einen geringen Beitrag zum Lernen. Das Ziel, sie adäquat $\mathrm{zu}$ verteilen und bereitzustellen, wurde in den letzten Jahrzehnten übererfüllt (Hesse, 2000). Obwohl eine Vielzahl von Informationen heute schnell und bequem zugänglich ist, gestaltet sich der Prozess des Wissenserwerbs unverändert mühsam. Lernportale sollten, nicht nur um ihrem Namen gerecht zu werden, diesen Prozess mit den genuinen Möglichkeiten computergestützter Didaktik unterstützen und nicht - das Internet als günstiges Distributionsmedium nutzend - die neuen Chancen ungenutzt lassen. 


\section{MedienPädagogik}

Die wirksame Unterstützung des Lernprozesses ist das Gütesiegel von Lernplattformen, auf das auch in Zukunft nicht verzichtet werden kann. Die Standardisierung ist dabei unverzichtbar - jedoch zur Unterstützung und nicht auf Kosten lernwirksamer didaktischer Konzeptionen.

\section{Literatur}

Anderson, J. R. (1989). Kognitive Psychologie: Eine Einführung (2. Aufl.). Heidelberg: Spektrum.

Daten + Dokumentation GmbH (2002). Glossar. Online unter http://www. daten-dokumentation.de/infos/glossar1.html

Fietkau, H.-J. (1994). Umweltspsychologie. In R. Ansanger \& G. Wenninger (Hrsg.), Handwörterbuch Psychologie (5. Aufl., S. 808-812). Weinheim: Belz.

Fromann, U. (2001). Workshop e-learning Plattformen 13./14. November 2001 in Köln. Online unter http://www.bi.fhg.de/PT-NMB/Bereich_Hochschulen/frommann_bericht_end

Gräsel, C., Prenzel, M. \& Mandl, H. (1993). Konstruktionsprozesse beim Bearbeiten eines fallbasierten Computerlernprogramms. In C. Tarnai (Hrsg.), Beiträge zur empirischen pädagogischen Forschung (S. 55-67). Münster: Waxmann.

Groen, J. G. \& Patel, V. L.(1991). A view from Medicin. In M. U. Smith (Hrsg.), Toward a Unified Theory of Problem Solving: Views From the Content Domain (S. 35-44). Hillesdale: Lawrence Erlbaum Associates.

Gruber, H. (1999). Mustererkennung und Erfahrungswissen. In M. Fischer \& W. Bartens (Hrsg), Zwischen Erfahrung und Beweis - medizinische Entscheidungsfindung und Evidence-Based Medicine (S. 25 - 52). Bern: Verlag Hans Huber

Hesse, F. W. (2000). Neue Konzepte für die Lehre unter den Bedingungen der neuen Medien. Beitrag zur Veranstaltungsreihe "Universitäten in der Wissensgesellschaft, http://www.wissensgesellschaft.org/themen/ bildung/ neuemedien.html

IEEE P1484.12/D6.1 (2001). Draft Standard for Learning Object Metadata. Sponsored by the Learning Technology Standardization Committee of the IEEE. Online unter http://www.math.tamucc.edu/ SCORM1_2/course/concepts/beginner/assets/ieeeLTSC.pdf

Kindt, M. (2001). Workshop e-learning Plattformen am 13. / 14. November in Köln. Workshop 3: Didaktische Szenarien und Einsatzstrategien. Online unter http://www.campussource.de/events/e0111koeln/ docu_e0111koeln/elearning_workshop3.pdf

Kolodner, J. L. (1983). Towards an understanding of the role of experience in the evolution from novice to expert. International Journal of ManMachine Studies, 19, 497 - 518.

Schank, R. C. (1994). Goal-Based Scenarios: A radical look at education. Journal of the Learning Sciences, 3, 429-453.

Schmidt H. G., Norman, G. R. \& Boshuizen, H. P. A. (1990). A cognitive perspective on medical expertise: theory and Implications. Academic medicine, 65, 611-621.

Schulmeister, R. (2001). Szenarien netzbasierten Lernens. In E. Wagner \& M. Kindt (Hrsg.), Virtueller Campus: Szenarien - Strategien - Studium (S. 16 - 38). Münster: Waxmann.

Schwan, S. \& Buder, S. (im Druck). Lernen und Wissenserwerb in Virtuellen Realitäten. In G. Bente (Hrsg.), Digitale Welten. Virtuelle Realität als Gegenstand und Methode der Psychologie. Göttingen: Hogrefe.

SCORM Resources (2001). Resources. Online unter http://www.math. tamucc.edu/SCORM1_2/resources.html

Tergan, S.-O. (1998). Lernen mit Hypermedien: Euphorie, Ernüchterung und Perspektiven. In E. Begander (Hrsg.), Über Wissen und Lernen (S. 1-14), Kolloquienreihe des AWM 1997/1998. Tübingen: Deutsches Institut für Fernstudienforschung.

Wiley, D. A. (2001). Connecting learning objects to instructional design theory: A definition, a metaphor, and a taxonomy. (S. 2, 29). Online: http://www.reusability.org/read/chapters/wiley.doc. 